Check for updates

Cite this: RSC Adv., 2017, 7, 54928

Received 17th August 2017

Accepted 17th November 2017

DOI: $10.1039 / \mathrm{c} 7 \mathrm{ra0} 9105 \mathrm{~b}$

rsc.li/rsc-advances

\title{
Indium-doped ZnO horizontal nanorods for high on-current field effect transistors
}

\author{
Ziqiang Zhu, (D) ab Borui Li, ${ }^{\text {b }}$ Jian Wen, ${ }^{\mathrm{b}}$ Zhao Chen, ${ }^{\mathrm{b}}$ Zhiliang Chen, $^{\mathrm{b}}$ Ranran Zhang, ${ }^{\mathrm{a}}$ \\ Shuangli Ye, ${ }^{a}$ Guojia Fang (D) *b and Jun Qian (D) *a
}

High on-current field effect transistors (FETs) are highly desirable for driving information displays such as active matrix organic light-emitting diode displays. Herein, indium-doped ZnO (IZO) horizontal nanorod arrays were fabricated for high on-current FETs by a facile and tunable hydrothermal method. We have found that indium doping can influence the growth behavior of $\mathrm{ZnO}$ nanorods. After indium doping, the $\mathrm{ZnO}$ nanorods tend to grow better along the horizontal direction and have a better flat morphology. More importantly, indium doping increases the carrier concentration of the IZO nanorods; this leads to better transfer and output performances of the IZO nanorod FETs. Therefore, the IZO nanorod FET with a high on-current of $6.39 \times 10^{-4} \mathrm{~A}$ and a field effect mobility of $26.3 \mathrm{~cm}^{2} \mathrm{~V}^{-1} \mathrm{~s}^{-1}$ has been synthesized and demonstrated in this study.

\section{Introduction}

In the past few decades, $\mathrm{ZnO}$ nanorods have attracted significant attention ${ }^{1}$ because of their unique photoelectrical properties $^{2-6}$ such as wide direct band-gap (3.37 eV), electron transport confinement, and tunable electrical property by doping. ${ }^{7-11}$ Therefore, they have been applied in ultraviolet photodetectors, ${ }^{12-16}$ field electron emission displays, ${ }^{17}$ and field effect transistors (FETs).$^{18-20}$ Hydrothermally grown ZnO nanorods have been used in FETs because of their low temperature growth, tunable growth behavior, and electrical property. ${ }^{21,22}$ However, generally, ZnO nanorod FETs exhibit the problem of low on-current, which affects their practical applications. Some efforts have been made to increase the on-current of lateral $\mathrm{ZnO}$ nanorod FETs, which mainly include two aspects: (1) optimization of horizontal ZnO nanorod growth between electrodes without vertical growth or radial growth, better contact to gate dielectrics, and better contact with $\mathrm{ZnO}$ nanorods and (2) increase of the $\mathrm{ZnO}$ nanorod number and better alignment of ZnO nanorods between source and drain electrodes. ${ }^{21-27}$ Researchers have made some progress in the optimization of ZnO nanorod growth behavior using a more effective passivation electrode and complex processing method. ${ }^{23,28}$ However, the ZnO nanorod FETs obtained usually have a low on-current of $\mu \mathrm{A}$. A smart structure has been designed for alignment of a greater number of $\mathrm{ZnO}$ nanorods between the source and drain electrodes ${ }^{27}$ however, it is not convenient for integration. Doping is an effective way to increase the carrier concentration

${ }^{a}$ School of Printing and Packaging, Wuhan University, Wuhan, 430072, P. R. China. E-mail:whuqianjun@163.com

${ }^{b}$ School of Physics and Technology, Wuhan University, Wuhan, 430072, P. R. China of semiconductors; however, to the best of our knowledge, it has not been used for on-current enhancement of horizontal $\mathrm{ZnO}$ nanorod FETs.

In this study, we have chosen indium doping for on-current enhancement of ZnO nanorod FETs because indium has an ionic radius similar to that of zinc and is less prone to oxidation. ${ }^{29-33}$ Interestingly, we found that In doping could influence the growth behavior of ZnO nanorods to some degree. Scanning electron microscopy (SEM) and transmission electron microscopy (TEM) analysis illustrate that indium doping benefits the horizontal growth of $\mathrm{ZnO}$ nanorods and results in a better flat morphology. The energy dispersive spectroscopy (EDS) and Xray photoelectron spectroscopy (XPS) analysis illustrate the presence of indium element in the $\mathrm{ZnO}$ nanorod samples. Electrical property measurement suggests that FETs obtained from indium-doped $\mathrm{ZnO}$ (IZO) nanorods have better performance than those obtained from undoped $\mathrm{ZnO}$ nanorods. The IZO nanorod FETs show a high on-current of $6.39 \times 10^{-4} \mathrm{~A}$ and a field effect mobility of $26.3 \mathrm{~cm}^{2} \mathrm{~V}^{-1} \mathrm{~s}^{-1}$.

\section{Experimental details}

For better comparison, FETs with both $\mathrm{ZnO}$ and IZO horizontal nanorods were synthesized under the same conditions. An $\mathrm{n}^{+}-\mathrm{Si}$ (resistivity $\sim 0.02 \Omega \mathrm{cm}$ ) wafer with a $240 \mathrm{~nm} \mathrm{SiO}_{2}$ layer was used as the starting substrate. The cut pieces were ultrasonically cleaned with acetone, ethanol, and deionized water, in sequence, and then dried using $\mathrm{N}_{2}$ gas. Photolithography was performed for defining the channel patterns of $5 \mu \mathrm{m}$ length and $50 \mu \mathrm{m}$ width. A $300 \mathrm{~nm}$ thick $\mathrm{ZnO}$ seed layer was deposited on the $\mathrm{SiO}_{2}$ substrate using a radio frequency magnetron sputtering deposition system, and then, a $70 \mathrm{~nm}$ thick tin-doped 
indium oxide (ITO) passivation layer was deposited. After a liftoff process with acetone, the patterned device structures of ITO $(70 \mathrm{~nm}) / \mathrm{ZnO}(300 \mathrm{~nm})$ on $\mathrm{SiO}_{2} / \mathrm{Si}$ substrates were obtained. For the hydrothermal process, an aqueous solution of zinc nitrate hexahydrate $\left(\mathrm{Zn}\left(\mathrm{NO}_{3}\right)_{2} \cdot 6 \mathrm{H}_{2} \mathrm{O}, 0.0025 \mathrm{M}\right)$ and equimolar hexamethylenetetramine (HMTA, $0.0025 \mathrm{M}$ ) was prepared, and the substrates were suspended upside down in the solution. The $\mathrm{ZnO}$ nanorod growth was performed in a sealed reaction vessel at $85{ }^{\circ} \mathrm{C}$ for $12 \mathrm{~h}$. For the IZO nanorod growth, the indium chloride tetrahydrate $\left(\mathrm{InCl}_{3} \cdot 4 \mathrm{H}_{2} \mathrm{O}\right)$ with a doping concentration of 2 at\% was added to the solution. All the reagents used in this synthesis were of analytical grade and used as-received without further purification. After the hydrothermal reaction, the fabricated samples were removed from the solution, rinsed with distilled water, and dried in an oven at $60{ }^{\circ} \mathrm{C}$ for $2 \mathrm{~h}$. Then, the FETs composed of horizontally aligned $\mathrm{ZnO}$ or IZO nanorods were obtained. The fabrication processes are illustrated in Fig. 1(a).

The growth morphologies and alignments of $\mathrm{ZnO}$ and IZO nanorods between source and drain electrodes were characterized using an SEM (Hitachi S-4800). The crystal property of the IZO nanorods was characterized using a TEM (JEM-2100). The crystal structure and growth orientation of the $\mathrm{ZnO}$ and IZO nanorods were characterized by an XRD (Bruker D8). The chemical composition of the IZO nanorod sample was characterized by an EDS analysis. The chemical bonding structures of $\mathrm{ZnO}$ and IZO nanorods were analyzed using an XPS (Thermo Scientific Escalab 250Xi). The electrical property of the $\mathrm{ZnO}$ and IZO nanorod FETs was characterized by measuring their transfer and output characteristics using a Keithley 4200 system.

\section{Results and discussion}

The schematic of the fabrication process of $\mathrm{ZnO}$ and IZO nanorod FETs is shown in Fig. 1(a). The in situ hydrothermal growth of $\mathrm{ZnO}$ nanorods proceeds with the $\mathrm{ZnO}$ seed layer and ITO passivation layer. The in situ hydrothermal growth of IZO nanorods proceeds under the same conditions, but with the In dopants in the solution. When grown between two square electrodes, the IZO nanorods assemble to form an FET. A representative optical image of the IZO nanorod array FETs is shown in Fig. 1(b). The IZO nanorods were uniformly grown and arranged well between electrodes.

Interestingly, In doping affects the growth behavior, including morphology and growth direction, of the ZnO nanorods, as shown in Fig. 2(a)-(f). Before In doping, the $\mathrm{ZnO}$ nanorods show a typical hexagonal prism morphology, which grow along both the horizontal and vertical directions. However, after In doping, the IZO nanorods show a belt morphology, which only grows along the horizontal direction. Moreover, different In doping contents affect the growth behavior of the ZnO nanorods. When the In doping content changes from 0.5 at $\%$ to 2 at\%, the bottom layer IZO nanorods sufficiently grow, but the top layer IZO nanorods gradually approach sufficient growth with an increase in the doping content. When the In doping content increases to 5 at\%, the arrangement begins to disperse. The In doping changes the surface energy of the IZO nanorods and affects the IZO nanorod growth to some degree. When the In doping content increases to $10 \mathrm{at} \%$, only sporadic IZO nanorods stretch out; this is probably attributed to the difficulty in increasing the nucleation and growth on the $\mathrm{ZnO}$ seed layer. Based on better growth along the horizontal direction and better belt morphology, we choose 2 at\% In doping content to fabricate IZO nanorod FETs in this study. The variation of growth behavior of IZO nanorods is beneficial for better contact with dielectrics and electrodes and carrier transport performance enhancement, which may result in electrical performance improvement of the IZO nanorod FETs.

For further observation, a high resolution transmission electron microscopy (HRTEM) image of the IZO nanorod is shown in Fig. 3(a). In the low magnification TEM image for the IZO nanorods in the inset of Fig. 3(a), we can find that the IZO nanorods show a belt morphology rather than the typical $\mathrm{ZnO}$ hexagonal prism morphology; this can be ascribed to the surface energy change of IZO nanorods with In doping. The wave-like edge could be ascribed to the instability of crystallization and etching on the surface of IZO nanorods in the reaction solution. The growth direction is along the $c$-axis (001) direction. The lattice spacing along the (001) direction is $0.52 \mathrm{~nm}$, corresponding to the lattice constant $c$ of the $\mathrm{ZnO}$ crystal. The selected area electron diffraction (SAED) image is shown in Fig. 3(b), suggesting the same abovementioned observation. The crystal property of the $\mathrm{ZnO}$ and IZO nanorods was characterized by XRD. As shown in Fig. 4, both the $\mathrm{ZnO}$ and

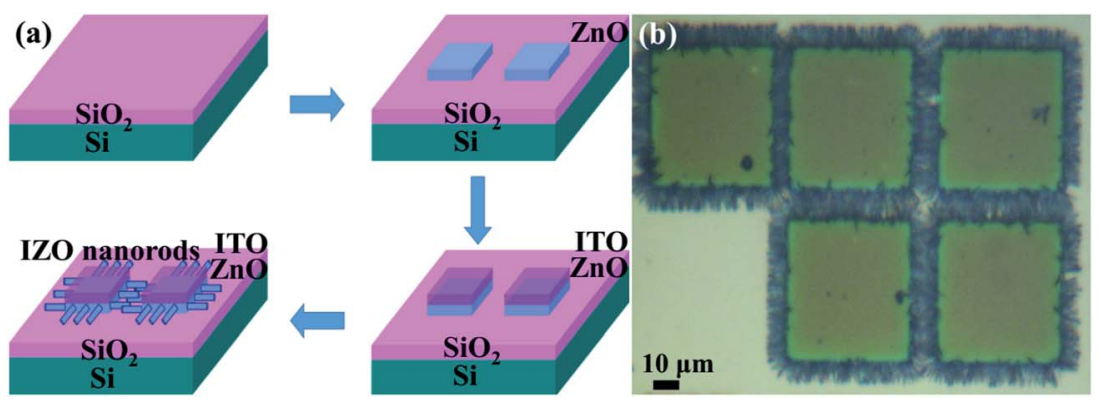

Fig. 1 (a) Schematic of the fabrication process and (b) optical image of the IZO nanorod FETs. 

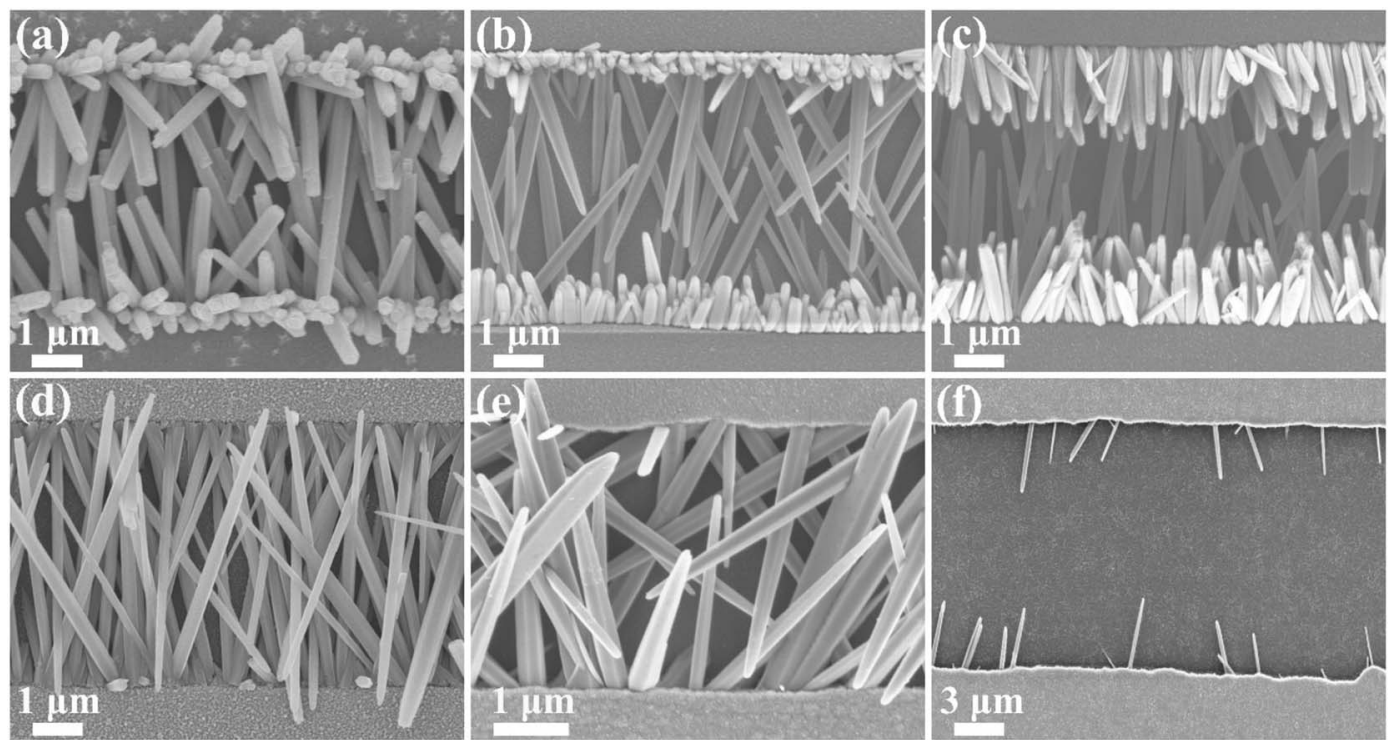

Fig. 2 SEM images of (a) undoped ZnO, (b) 0.5 at\%, (c) 1 at\%, (d) 2 at\%, (e) 5 at\%, and (f) 10 at\% In-doped ZnO nanorods.
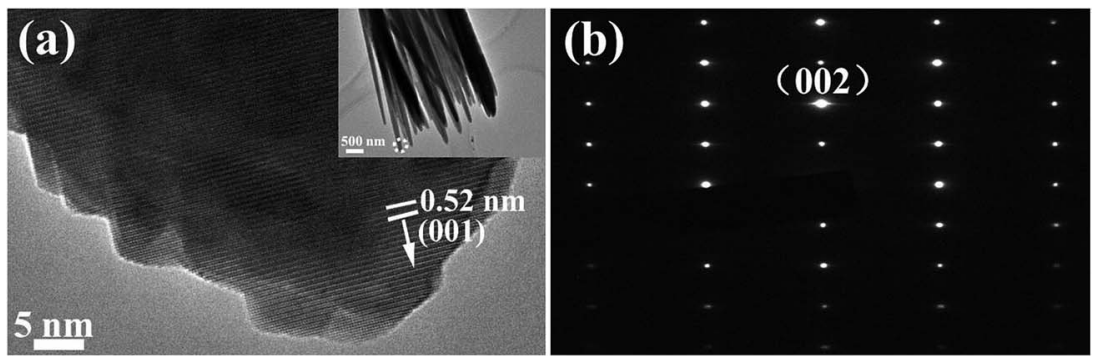

Fig. 3 (a) An HRTEM image of the IZO nanorod (the inset shows a low magnification image of the IZO nanorods), and (b) a SAED image of the IZO nanorod.

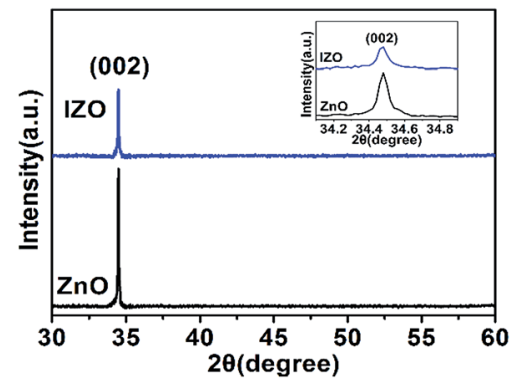

Fig. 4 XRD patterns of the $\mathrm{ZnO}$ and IZO nanorods on the $\mathrm{ZnO}$ seed layer.

IZO nanorods only show a strong characteristic peak of the (002) plane of the $\mathrm{ZnO}$ wurtzite structure. The peak position does not change after indium doping. We conclude that highly oriented nanorod arrays have been synthesized for both pure $\mathrm{ZnO}$ and indium-doped $\mathrm{ZnO}$ nanorod situations.

Fig. 5(a) shows the EDS spectrum of the IZO nanorods. Besides $\mathrm{Zn}, \mathrm{O}$, and Si peaks, an In peak was found. The indium content in IZO is about 0.45 at\%. The EDS spectrum shows that In is doped into the ZnO nanorods. Based on the EDS element mapping analysis of the IZO nanorod, as shown in Fig. 5(b), we can find only sporadic In element distribution in the nanorod.

To further confirm the doping of indium into IZO nanorods, XPS analysis was performed. The In $3 \mathrm{~d}$ spectrum of IZO nanorods is shown in Fig. 6(a). The appearance of the In spectrum and the absence of the $\operatorname{In}_{2} \mathrm{O}_{3}$ phase in the previous XRD patterns suggest that In element is doped into the $\mathrm{ZnO}$ crystal. In addition, compared to the $\mathrm{ZnO}$ nanorod sample, the $\mathrm{Zn} 2 \mathrm{p}_{3 / 2}$ and $\mathrm{Zn} 2 \mathrm{p}_{1 / 2}$ peaks of IZO nanorods shift slightly towards a higher binding energy (as shown in Fig. 6(b)). The peak shifts can be explained by the difference in the electronegativity of In $(\chi=1.78)$ and $\mathrm{Zn}(\chi=1.65)$. Because of the higher electronegativity of In than that of $\mathrm{Zn}$, the valence electron density of $\mathrm{Zn}$ in the $\mathrm{Zn}-\mathrm{O}-\mathrm{In}$ bond in the IZO nanorod sample becomes lower than that in the $\mathrm{Zn}-\mathrm{O}-\mathrm{Zn}$ bond in the $\mathrm{ZnO}$ nanorod sample. Consequently, the screening effect of $\mathrm{Zn}$ in the IZO nanorod sample is weakened, and the binding energy of $\mathrm{Zn} 2 \mathrm{p}$ increases. The $\mathrm{O} 1 \mathrm{~s}$ deconvoluted spectra of the $\mathrm{ZnO}$ and IZO nanorods are shown in Fig. 6(c). The low binding energy peak $\left(\mathrm{O}_{\mathrm{I}}\right)$ corresponds to $\mathrm{O}^{2-}$ combined with metal ions in the $\mathrm{ZnO}$ and IZO crystals. The medium binding energy peak $\left(\mathrm{O}_{\text {II }}\right)$ is related to the oxygen deficiency in the $\mathrm{ZnO}$ and IZO nanorods 

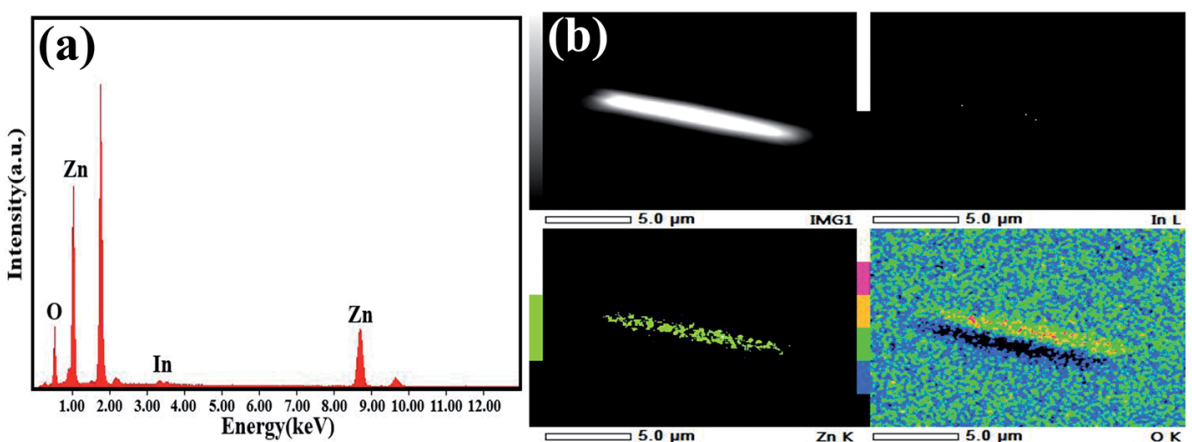

Fig. 5 (a) EDS spectrum and (b) EDS element mapping analysis of the IZO nanorod.
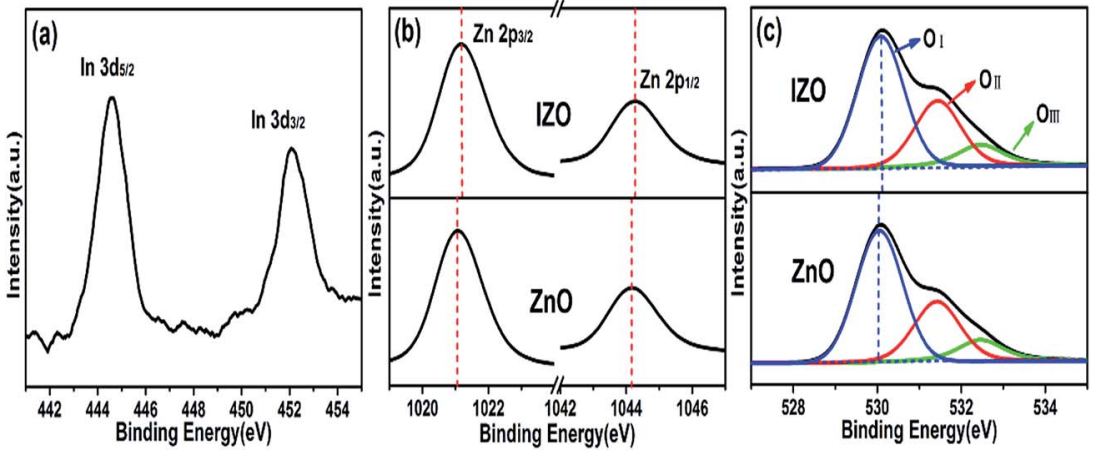

Fig. 6 XPS spectra of the $\mathrm{ZnO}$ and IZO nanorods corresponding to (a) $\ln 3 \mathrm{~d}$, (b) $\mathrm{Zn} 2 \mathrm{p}$, and (c) O 1s.

crystals, indicating the corresponding oxygen vacancy concentration. The high binding energy peak $\left(\mathrm{O}_{\mathrm{III}}\right)$ is ascribed to some chemisorbed oxygen components such as $-\mathrm{CO}_{3}$ and $\mathrm{H}_{2} \mathrm{O}$. We can find that the $\mathrm{O}$ 1s peak of the IZO nanorods also shifts slightly towards a higher binding energy as compared to that of the undoped $\mathrm{ZnO}$ nanorod sample. The shift is due to the higher electronegativity of In ion than that of $\mathrm{Zn}$ ion, as abovementioned. An intensity ratio of $\mathrm{O}_{\mathrm{II}} / \mathrm{O}_{\text {total }}$ is obtained to compare the relative concentration of the oxygen vacancies: the values are 0.29 and 0.31 for the $\mathrm{ZnO}$ and IZO nanorod samples,
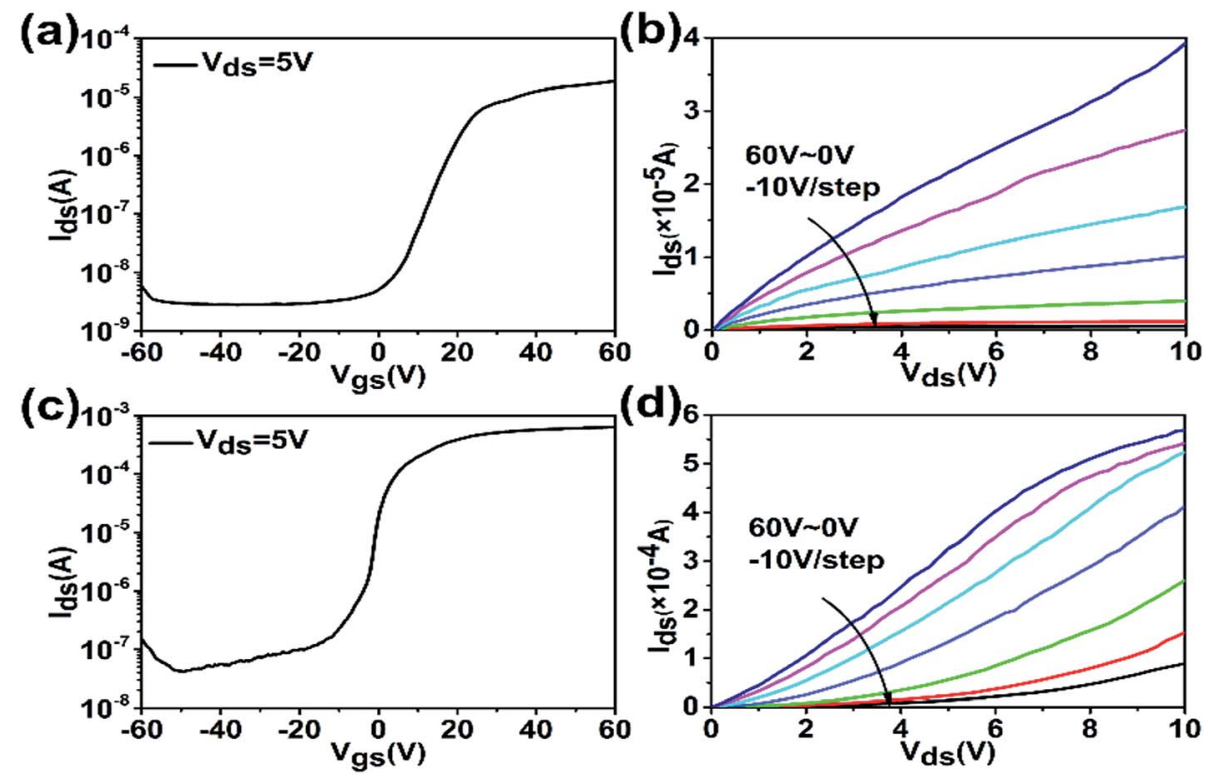

Fig. 7 (a) Transfer characteristic and (b) output characteristic of the ZnO nanorod FETs. (c) Transfer characteristic and (d) output characteristic of the IZO nanorod FETs. The measurements were conducted under the same conditions as shown in the figure. 
Table 1 Transistor characteristic parameters of $\mathrm{ZnO}$ and IZO nanorod FETs

\begin{tabular}{lccll} 
& $\mu$ & & & \\
& $\left(\mathrm{cm}^{2} \mathrm{~V}^{-1} \mathrm{~s}^{-1}\right)$ & $V_{\text {th }}(\mathrm{V})$ & On/off ratio & On-current (A) \\
\hline ZnO & 1.06 & 10.99 & $10^{3}$ & $1.86 \times 10^{-5}$ \\
IZO & 26.3 & -3.32 & $10^{3}$ & $6.39 \times 10^{-4}$
\end{tabular}

respectively. The IZO nanorod sample with a higher value has more oxygen vacancies than $\mathrm{ZnO}$ because of the lower bond strength of In-O as compared to that of $\mathrm{Zn}-\mathrm{O}$. On the basis of the abovementioned analysis results, the In element is doped into the $\mathrm{ZnO}$ crystal, which would generate more oxygen vacancies; therefore, a higher carrier concentration can be obtained for the IZO nanorod arrays.

The transfer characteristic curve of the $\mathrm{ZnO}$ nanorod FETs is shown in Fig. 7(a). The ZnO nanorod FETs can be well controlled by the gate voltage and exhibit a typical n-type transistor behavior. The field effect mobility of the horizontal nanorod array FETs is approximately calculated by $\mu_{\mathrm{FE}}=L g_{\mathrm{m}} /$ $W C_{\mathrm{i}} V_{\mathrm{ds}}$. The mobility obtained from the $\mathrm{ZnO}$ nanorod array FET is $1.06 \mathrm{~cm}^{2} \mathrm{~V}^{-1} \mathrm{~s}^{-1}$, and the on/off ratio is $10^{3}$. The on-current of ZnO nanorod FETs is $1.86 \times 10^{-5} \mathrm{~A}$ at $V_{\mathrm{gs}}=60 \mathrm{~V}$ and $V_{\mathrm{ds}}=5 \mathrm{~V}$, which cannot meet practical requirement and still needs to be improved. The threshold voltage of the ZnO nanorod FETs is $10.99 \mathrm{~V}$. The output characteristics curve of the $\mathrm{ZnO}$ nanorod FETs in Fig. 7(b) shows a distinct linear region, and there is no saturation current region when $V_{\mathrm{ds}}$ is swept from 0 to $10 \mathrm{~V}$ and $V_{\mathrm{gs}}$ varies from 0 to $60 \mathrm{~V}$ with a $10 \mathrm{~V}$ per step. After In doping, the electrical property variation of the nanorod FETs is shown in Fig. $7(\mathrm{c})$ and (d). When the $V_{\mathrm{gs}}$ varies from $-40 \mathrm{~V}$ to $-60 \mathrm{~V}$, there is an upward trend, as shown in Fig. 7(c). This could be due to the formation of an inversion channel. The mobility obtained from the IZO nanorod array FETs is $26.3 \mathrm{~cm}^{2} \mathrm{~V}^{-1} \mathrm{~s}^{-1}$, and the on/off ratio is $10^{3}$. The on-current of IZO nanorod FETs is $6.39 \times$ $10^{-4} \mathrm{~A}$ at $V_{\mathrm{gs}}=60 \mathrm{~V}$ and $V_{\mathrm{ds}}=5 \mathrm{~V}$, which has been enhanced by an order of magnitude because of slight In doping. The threshold voltage of IZO nanorod FETs is $-3.32 \mathrm{~V}$, which is decreased as compared to that of the ZnO nanorod FETs. The output characteristic curve of the IZO nanorod FETs in Fig. 7(d) shows a current-voltage behavior similar to that of $\mathrm{ZnO}$ nanorod FETs, but the $I_{\mathrm{ds}}$ of IZO nanorod FETs is larger than that of the $\mathrm{ZnO}$ nanorod FETs. The characteristic parameters of $\mathrm{ZnO}$ and IZO nanorod FETs are summarized in Table 1. As a result, the In doping could increase oxygen vacancy concentration, carrier concentration, and field effect mobility. The FET sourcedrain current has been enhanced to quite a high level because of In doping. The IZO horizontal nanorod array FETs may have potential applications for future large current power devices.

\section{Conclusions}

In summary, we have successfully fabricated horizontally grown IZO nanorod arrays and the corresponding FETs by a simple hydrothermal method. EDS and XPS analyses illustrate that there is In element in the nanorod array samples. We have found that In doping affects both growth behavior and morphology of the $\mathrm{ZnO}$ nanorods. After In doping, the $\mathrm{ZnO}$ nanorods tend to grow along the horizontal direction and have a better flat morphology. After In doping, more oxygen vacancies are produced, and the electrical property of IZO nanorod FETs is improved. The IZO nanorod FETs show a high oncurrent of $6.39 \times 10^{-4} \mathrm{~A}$ at $V_{\mathrm{ds}}=5 \mathrm{~V}$ and $V_{\mathrm{gs}}=60 \mathrm{~V}$ and a field effect mobility of $26.3 \mathrm{~cm}^{2} \mathrm{~V}^{-1} \mathrm{~s}^{-1}$. The IZO horizontal nanorod FETs may have potential applications for large driving current power devices.

\section{Conflicts of interest}

There are no conflicts to declare.

\section{Acknowledgements}

This work was supported by the Hubei Science and Technology Support Project (No. YJG0261), the Wuhan Science and Technology Research Projects (No. 2014010101010002), and the National Natural Science Foundation of China (No. 11674252, No. 61376013).

\section{References}

1 K. P. Jayadevan and T. Y. Tseng, J. Nanosci. Nanotechnol., 2012, 12, 4409-4457.

2 X. Peng, Y. Zeng, X. Pan, W. Wang, Y. Zhou, F. Wang, Q. Lu and Z. Ye, RSC Adv., 2017, 7, 29440-29445.

3 Z. Liu, P. Kuang, R. Wei, N. Li, Y. Chen and Y. Su, RSC Adv., 2016, 6, 16122-16130.

4 X. Zou, G. Fang, J. Wan, N. Liu, H. Long, H. Wang and X. Zhao, Semicond. Sci. Technol., 2011, 26, 1-5.

5 R. Zhu, W. Zhang, C. Li and R. Yang, Nano Lett., 2013, 13, 5171-5176.

6 Z. Wang, R. Yang, J. Zhou, Y. Qin, C. Xu, Y. Hu and S. Xu, Mater. Sci. Eng., R, 2010, 70, 320-329.

7 D. Bi, G. Boschloo, S. Schwarzmuller, L. Yang, E. J. Johansson and A. Hagfeldt, Nanoscale, 2013, 5, 11686-11691.

8 C. Hsu and S. Chang, Small, 2014, 10, 4562-4585.

9 G. C. Park, S. M. Hwang, J. H. Choi, Y. H. Kwon, H. K. Cho, S. W. Kim, J. H. Lim and J. Joo, Phys. Status Solidi A, 2013, 210, 1552-1556.

10 K. Mahmood, S. B. Park and H. J. Sung, J. Mater. Chem. C, 2013, 1, 3138-3149.

11 E. Fortunato, P. Barquinha and R. Martins, Adv. Mater., 2012, 24, 2945-2986.

12 Y. Zeng, X. Pan, W. Dai, Y. Chen and Z. Ye, RSC Adv., 2015, 5, 66738-66741.

13 H. Zhou, P. Gui, Q. Yu, J. Mei, H. Wang and G. Fang, J. Mater. Chem. C, 2015, 3, 990-994.

14 Z. Chen, B. Li, X. Mo, S. Li, J. Wen, H. Lei, Z. Zhu, G. Yang, P. Gui, F. Yao and G. Fang, Appl. Phys. Lett., 2017, 110, 123504.

15 N. Liu, G. Fang, W. Zeng, H. Zhou, F. Cheng, Q. Zheng, L. Yuan, X. Zou and X. Zhao, ACS Appl. Mater. Interfaces, 2010, 2, 1973-1979. 
16 B. Sven, H. R. Francisco, J. D. Holmes and R. R. Albert, Prog. Mater. Sci., 2010, 55, 563-627.

17 N. Liu, G. Fang, W. Zeng, H. Long, X. Fan, L. Yuan, X. Zou, Y. Liu and X. Zhao, J. Phys. Chem. C, 2010, 114, 8575-8580.

18 R. Ahmad, N. Tripathy, M. Y. Khan, K. S. Bhat, M. S. Ahn and Y. B. Hahn, RSC Adv., 2016, 6, 54836-54840.

19 P. B. G. Conor and O. V. P. Natalie, J. Vac. Sci. Technol., B: Nanotechnol. Microelectron.: Mater., Process., Meas., Phenom., 2013, 31, 06F101.

20 W. I. Park, J. S. Kim, G. C. Yi, M. H. Bae and H. J. Lee, Appl. Phys. Lett., 2004, 85, 5052-5054.

21 S. Xu and Z. Wang, Nano Res., 2011, 4, 1013-1098.

22 Y. Hahn, Korean J. Chem. Eng., 2011, 28, 1797-1813.

23 Y. Qin, R. Yang and Z. Wang, J. Phys. Chem. C, 2008, 112, 18734-18736.

24 B. Weintraub, Z. Zhou, Y. Li and Y. Deng, Nanoscale, 2010, 2, 1573-1587.

25 J. Wang, P. Yang, T. Hsieh, C. Hwang, D. Shye and I. Lee, Solid-State Electron., 2012, 77, 72-76.
26 T. Demes, C. Ternon, F. Morisot, D. Riassetto, M. Legallais, H. Roussel and M. Langlet, Appl. Surf. Sci., 2017, 410, 423431.

27 Y. K. Park, W. Y. Rho, T. Mahmoudi and Y. B. Hahn, Chem. Commun., 2014, 50, 10502-10505.

28 Y. K. Park, H. S. Choi, J. H. Kim, J. H. Kim and Y. B. Hahn, Nanotechnology, 2011, 22, 1-7.

29 M. Ahmad, H. Sun and J. Zhu, ACS Appl. Mater. Interfaces, 2011, 3, 1299-1305.

30 J. Xiao, X. Hou, L. Zhao and Y. Li, Int. J. Hydrogen Energy, 2016, 41, 14596-14604.

31 D. P. Norton, Y. W. Heo, M. P. Ivill, K. Ip, S. J. Pearton, M. F. Chisholm and T. Steiner, Mater. Today, 2004, 7, 34-40. 32 S. Chang, B. Duan, C. Liu, C. Hsiao, S. Young and C. Huang, IEEE Trans. Electron Devices, 2013, 60, 3901-3906.

33 C. E. Benouis, M. Benhaliliba, A. S. Juarez, M. S. Aida, F. Chami and F. Yakuphanoglu, J. Alloys Compd., 2010, 490, 62-67. 